\title{
Preparation of Continually Graded Barriers of YPrBaCuO for HTS Josephson Junctions
}

\author{
I. Gao, J.L. Sun, and W.H. Tang
}

\begin{abstract}
We report preparation of a novel barrier structure for high $T_{t}$ superconducting multilayer Josephson junctions using a simple composite target technique. Such a barrier consists of $\mathrm{Y}_{x} \mathrm{Pr}_{1-x} \mathrm{Ba}_{2} \mathrm{Cu}_{3} \mathrm{O}_{4}$ with a continually varied concentration of yttrium. In this harrier no lattice mismatch and other incompatibility problems occur between adjacent layers. Thus the formation of interfacial defects and structural strain can be mostly prevented. The Josephson coupling takes place at the electrically formed interfaces rather than the structural interfaces. A particular feature of these junctions is that the effective thickness of the barrier strongly depends on the measuring temperature and the concentration gradient. The absence of the structural interface in the weak link region greatly enhances the refroducibility and the performance of these junctions.
\end{abstract}

Index Terms-Hligh Temperature Superconductors, Josephson Junctions

\section{INTRODUCTION}

$\mathrm{O}$ ne of the major problems associated with high $T_{c}$ Josephson junctions using an artificial barrier is the poor interface between the barrier and superconductor. It is believed that the interfaces between barrier and superconducting electrodes play a crucial role. $[1-4]$ In general the interface between the bartier and superconductors is damaged and defective, which results in poor performance and reproducibility. Studies using a high resolution transmission electron microscope (HRTEM) showed that the structural, thermal, and chemical incompatibility at the interface could introduce various defects.[5-6] Also, the ex situ lithography process involved by either ion-milling or shemical etching further damage the interface.[1,7-9] To reduce the incompatibility between barrier and electrode, and to improve the damaged interface, various approaches like chemical etching and use of intermediate layers were reported. $\{7,9\}$ However, the interfacial problems still remain although the quality of the interfaces or the lattice mismatch between barrier

Manuscript received September 16,2000. This work has been supported by the Rescatch Grants Council (RGC) of Hong Kong.

3. Gao is with Department of Physics, The Univensity of Hong Kong Pokfulam Roul, Hong Kong (telephone: 852-28597948, fax: 852-25599152, e-mail: jngao(ahku.hk).

I.L. Sun is with Department of Physics, The University of Hong Kong, Pokfulam Road, Hong Kong:

W.H. Tang is with Deparment of Physics, The Lniversity of Hong Kong Pokfulam Road, Hong Kong (telephone: 852-28592195, lax: 852-25599152, e-mail: whtang(ahlu.hk). and superconductor could be improved. The reproducibility and performance of high $T_{\mathfrak{c}}$ multilayer junctions are still not satisfactory.

In this paper, we describe tle preparation of a new barrier structure with a continually graded composition of $\mathrm{Y}_{\mathrm{x}} \mathrm{Pr}_{1-\mathrm{x}} \mathrm{Ba}_{2} \mathrm{Cu}_{3} \mathrm{O}_{\mathrm{S}^{\prime}}\left(\mathrm{Y}_{\mathrm{x}} \mathrm{Pr}_{1 \times \mathrm{x}} \mathrm{BCO}\right)$. High $\mathrm{T}_{\mathrm{t}}$ Josephson junctions with such a graded barrier structure have no structural interfaces and the Josephson coupling takes place at the electronically formed interfaces. In addition, the effective barrier thickness in our junctions, which depends on the temperature and concentration gradient, can be varied. Thus the reproducibility and uniformity of these junctions are greatly enhanced.

\section{PREPARATION OF THE GRADED BARRIER AND JUNCTTONS}

Our junctions were fabricated on $\mathrm{SrTiO}_{3}$ (STO) substrates by using off-axis RF magnetron sputtering. First, an epitaxial bi-layer consisting of the base $\mathrm{YBa}_{2} \mathrm{Cu}_{3} \mathrm{O}_{2}(\mathrm{YBCO})$ electrode and the insulating layer of $\mathrm{PrBa}_{2} \mathrm{Cu}_{3} \mathrm{O}_{y}$ (PBCO) was grown in situ using off-axis sputtering. Photoresist was then spun onto the bi-layer films ats the stencil and was baked at $300^{\circ} \mathrm{C}$ for 10 min. The structure was patterned by a standard lithographic process. To create a ramp edge, the samples with paiterned resist were ion milled through the bi-layer and slightly into the substrate by using a Katufinann Ar ion beam gun of $5 \mathrm{~cm}$ in diameter. The ion milling was performed under a pressure of (5-8) $\times 10^{-4} \mathrm{mbar}$, and the beam current and voltage were $I=15$ $\mathrm{mA}$ and $V=600-800 \mathrm{~V}$, respectively. 'To prevent overheating of the sample, the ion gun was operated in a pulse mode, which allowed a short break every 10 seconds. After the ramp edge being made, the sample was placed back into the vacuum chamber and the barrier and top electrode were then grown.

To overcome the problem caused by the damaged interface, $\mathrm{Y}_{2} \mathrm{Pr}_{1-x} \mathrm{BCO}$ was used as the barrier with the concentration $x$ gradually changing from 1 to 0 and then from 0 to 1 , as shown in Fig. 1. We first used two targets co-spultering to make such a continually graded barrier of $\mathrm{Y}_{x} \mathrm{Pr}_{1-x} \mathrm{BCO}$. By controlling the sputter power of $\mathrm{YBCO}$ and $\mathrm{PBCO}$ targets, a continuous composition gradient could be formed. The drawbacks of this method are that the gradient of concentration $x$ is not very uniform and the deposition system is very complicated. Moreover, it is quite difficult to control the process to obtain a smoothly clianged composition in the grown barrier. Therefore we developed a composite target technicue for preparing the graded barrier. A schematic diagram of our deposition system is shown in Fig. 2. The composite target consists of two semi-dises, YBCO and $\mathrm{PBCO}$, respectively. It was made 
simply by placing YBCO and PBCO powder in the mould, each occupying a half volume, and then pressing into a disc. Il is known that the YBCO and PBCO can be sputtered under the same conditions with a similar rate. At room temperature the resistance of $\mathrm{YBCO}$ and $\mathrm{PBCO}$ are in the same order and the sputtering was operated in RF-mode. Thus the sputter power could be divided more or less equally into the two sites of the composite target, as can be seen from the shape of the sputter plasina. During the growth of barrier, the substrate was slowly rotated from the $\mathrm{YBCO}$ side to the $\mathrm{PBCO}$ side and then to the $\mathrm{PBCO}$ target to make a pure $\mathrm{PBCO}$ central layer. The reason that we insert a $\mathrm{PBCO}$ central layer between two graded $\mathrm{Y}_{\mathrm{x}} \mathrm{Pr}_{1-\mathrm{x}} \mathrm{BCO}$ layers is to ensure that no short circuits can take place even at low temperatures. After the $\mathrm{PBCO}$ barrier being deposited, the substrate is rotated back to the compound $\mathrm{YBCO} / \mathrm{PBCO}$ target and gradually moved from $\mathrm{PBCO}$ side to YBCO side, and then to the YBCO target to grow the top electrode. By using such a simple technique, a smooth changing of the composition from $\mathrm{YBCO}$ to $\mathrm{PBCO}$ is obtained. A continually graded barrier without any abrupt clianges in structure and composition is therefore formed.

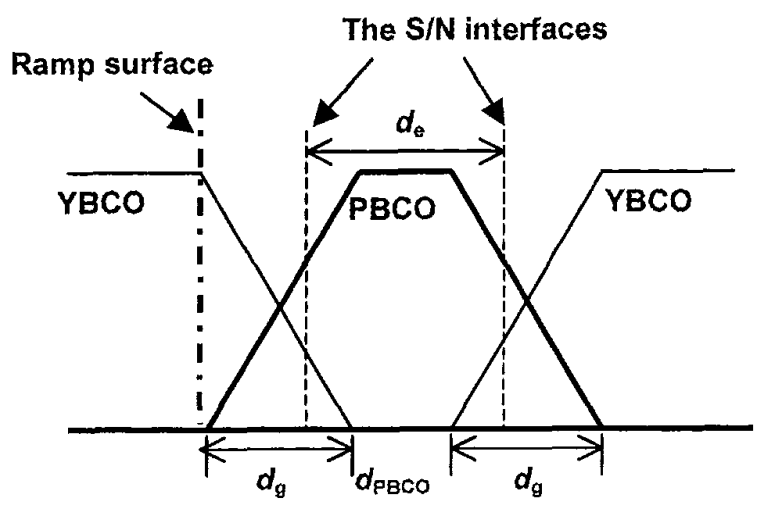

Fig. I Schematic diagram of the continually graded barrier of $\mathrm{Y}_{\mathrm{X}} \mathrm{Pr}_{1-\mathrm{X}} \mathrm{Ba}_{2} \mathrm{Cu}_{3} \mathrm{O}_{7}$ with the concentration. $\mathrm{r}$ gradually changing from 1 to 0 and then from 0 to $1, d_{x}$ is the effective barrier thickuess.

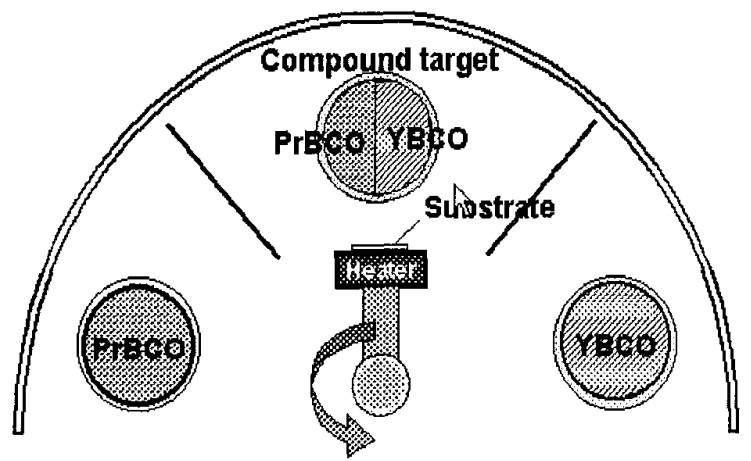

Fig2. Schematic diagram of our deposition system.

\section{RESULTS AND DISCUSSION}

Fig. 3 presents the depth distribution of yttrium and praseodymium measured by $x$-ray photoelectron spectrometry (XPS). During the measurement, the film was sputtered through the top YBCO layer, the graded $\mathrm{Y}_{\mathrm{x}} \mathrm{Pr}_{1 \times x} \mathrm{BCO}$ barrier, the bottom YBCO layer, and into the $\mathrm{SrTiO}_{3}$ substrate. As can be seen in Fig. 3, the yttrium concentration $x$ was gradually changed from $x=1$ to $x=0$ to reach a pure $\mathrm{PBCO}$ barrier, and then $x$ was slowly increased from 0 to $I$ to reach the YBCO electrode. Therefore the mismatching between every adjacent atomic layer would be very small and the occurrence of interface defects can be greatly reduced. Hence the reproducibility and performance of these junctions can be signilicantly enhanced. On the XISS spectrum the ytrium signal did not reach zero level at the center of barrier although there was a pure $P B C O$ central layer in the barrier. The reason could be due to the penetration of the probe through the entire $\mathrm{PBCO}$ layer into the bottom YBCO layer as the probe depth in XPS is typically $10 \mathrm{~nm}$.

In the ramp-type junctions using a conventional single PBCO barrier, the ramp surface created by ion milling is typically defective. The damaged interface can result in a large boundary resistance and low transparency for the quasi-particles at the interface. $[9,10]$ Also, an abrupt change of lattice constant and composition at the interface can introduce strain and interfacial defects. [11] Thus the interface resistance rather than the barrier material could dominate the junction properties. In comparison, our junctions with a continuously graded barrier can mostly prevent the influence of the damaged ramp interface. It is known that the $Y_{x} \operatorname{Pr}_{1-x}$ BCOCan be a superconductor at sufficiently low temperature, if the yttrium concentration $x$ is larger than 0.5 . The results obtained on bulk material of $\mathrm{Y}_{\lambda} \mathrm{Pr}_{1-x} \mathrm{BCO \text {indicated }}$ that the transition temperature $T_{\mathrm{E}}$ decreased gradually as the concentration $x$ decreased. [12]

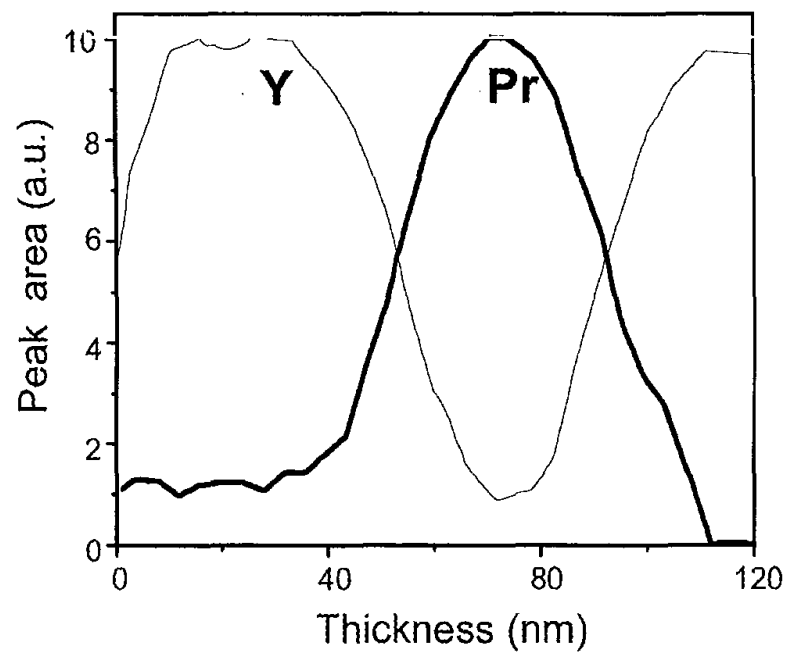

Fig. 3 The depth distribution of $Y$ and $P r$ measured by XPS. 


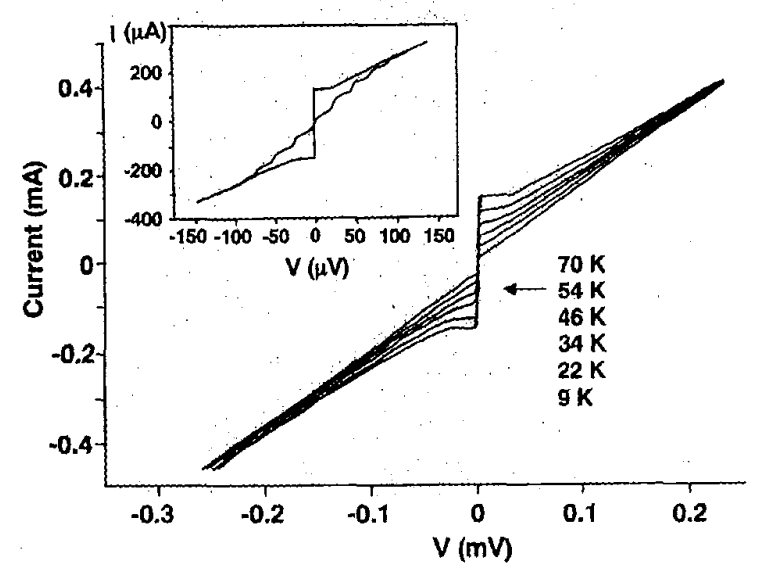

Fijg. $4 \quad I-F$ curves for junction with a $\mathrm{Y}_{x} \mathrm{P}_{1-\mathrm{x}} \mathrm{BCO}$ barrier at difierent temperatures $\left(d_{0}=30 \mathrm{~nm}\right.$ and $d$ enco $\left.\% 4 \mathrm{~nm}\right)$. The inset shows the $6-V$ curves with and withoul microwave radiation at $9 \mathrm{~K}$.

Depending on the concentration $x$ and $T$, the $\mathrm{Y}_{x} \operatorname{Pr}_{1-x} \mathrm{BCO}$ layer is partially superconductive. Therefore the $S / I$ interface will be formed inside the $Y_{x} P_{1-x} B C O$ layer and the damaged ramp surface is excluded from the weak link region, as shown in Fig. 1 . Since the concentration $x$ varies very smoothly there will be no abrupt change in the lattice constant and the composition. Hence nos structural interface is formed within the weak link region. To assess the influence of the damaged ramp surface, which now is located within the superconducting electrode, we deposited an YBCO layer on the damaged ramp surface. It was found that the film still showed a full transition with a high $T_{c}$. We conclude that the ramp surface within the superconducting region has little effect on the performance of the junctions.

The junctions with such a graded barrier present resistively shunted junction (RSI) characteristics. $I-V$ curves have small excess currents (see Fig.4). Almost all of these junctions exhibited clear Shapiro sleps under irradiation of a microwave lield, giving unambiguous evidence of the Josephson behavior. The junctions discussed in this paper were structured into a width of either 10 or $20 \mu \mathrm{m}$. The normal state resistance near the transition temperature in these junctions is typically from $10^{-1}$ to a few Ohm. The characteristic voltages, $I_{c} R_{\mathrm{m}}$, measured at $10 \mathrm{~K}$ are around $1 \mathrm{mV}$.

Using such a continuously graded barrier structure, the yield of successful junctions, which show a supercurrent and clear Shapiro steps, is nearly 100\% (Among 40 junctions made so far, 38 exhibited clear Shapiro steps above 50 K.). In contrast, the yield of good junctions with a single barrier is typically below $50 \%$. The uniformity of junction parameters, known as an essential issue for applications, was considered too. Damaged interfaces often cause variation in the junction properties like $I_{\mathrm{c}}$ and $R_{\mathrm{n}}$, etc. In most bigh $T_{\mathrm{c}}$ junctions with an artificial barrier, uniformity has been influenced by the damaged interfaces leading to large variation. By using this barrier, the damaged interface is excluded fiom the weak link region so it can minimally affect junction performance. In our junction process, $I_{\mathrm{c}}$ variation as low as $1-\sigma-12 \%$ for 10 junctions with identical parameters was found at 60-65 K.
A particular feature of such a barrier is that the effective barrier thickness $d_{\mathrm{e}}$, which is the spacing between the two $\mathrm{S} / \mathrm{N}$ interfaces (see Fig. 1), could be varied even after fabrication. In our junction the barrier consists of three layers, the graded $\mathrm{Y}_{\mathrm{A}} \mathrm{Pr}_{1 \times \mathrm{x}} \mathrm{BCO}$ interlayer, $\mathrm{PBCO}$ layer, and another graded interlayer. The graded intermediate layers with thickness $d_{\mathrm{g}}$ can be partially superconducting depending on the temperature and the concentration $x$. For most samples studied in this work, the thickness of the PBCO central layer is $d_{\mathrm{PBCO}}=8 \mathrm{~nm}$, and the thickness of the graded layer at the two sides was varied from $d_{\mathrm{g}}=6 \mathrm{~nm}$ to $d_{\mathrm{g}}=38 \mathrm{~nm}$. The effective barrier thickness should be the sum of $d_{p 13 c o}$ and the thickness of superconducting part within $Y_{X} \operatorname{Pr}_{1-X} \mathrm{BCO}$, which is a function of the remperature and the concentration gradient. It follows that the barrier thickness in such junctions would strongly depend on the measuring temperature.

Since the $d_{\mathrm{c}}$ varies as a function of temperature, it is therefore of great interest to study the temperature dependence of the transport properties in these junctions. The normal state resistance $R_{1} A$ ( $A$ is the junction area) is plotted in Fig. 5 against temperature. We found that for small values of $d_{\mathrm{g}}$ the $R_{\mathrm{n}} A$ products were quite insensitive to the temperature. It is known that $\mathrm{PBCO}$ is a semiconductor, whereas $\mathrm{Y}_{x} \mathrm{Pr}_{1-x} \mathrm{BCO}$ can behave as a normal metal, depending on the concentration $x$ and the temperature. As the effective barrier thickness is also changed, the whole barrier presents a complicated system. The temperature insensitive $R_{\mathrm{r}} A$ product is a result of the combination of $\mathrm{Y}_{x} \mathrm{Pr}_{1-x} \mathrm{BCO}$ and $\mathrm{PBCO}$ layers. On the other hand, with a thicker $\mathrm{Y}_{\mathrm{x}} \mathrm{Pr}_{1-x} \mathrm{BCO}$ layer $R_{\mathrm{r}} A$ is dominated by the $\mathrm{PBCO}$ material, which usually shows a semiconductor behavior.

The temperature dependence of $t_{c}$ of these junctions was also measured and compared with that of junctions with a conventional single barrier of PBCO. Fig. 6 shows the normalized $I_{c}$ as a function of reduced temperature $T / T_{6}$ for junctions with various $d_{\mathrm{g}}$ (from $10 \mathrm{~nm}$ to $38 \mathrm{~nm}$ ). Most of the data lie on a nearly straight line, approach a $\left(1-T / T_{c}\right)^{3}$ relationship. In contrast the $I_{e}$ of junctions using a single $\mathrm{PBCO}$ barrier usually exhibits a $\left(1-T / T_{c}\right)^{2}$ dependence of a SNS-type

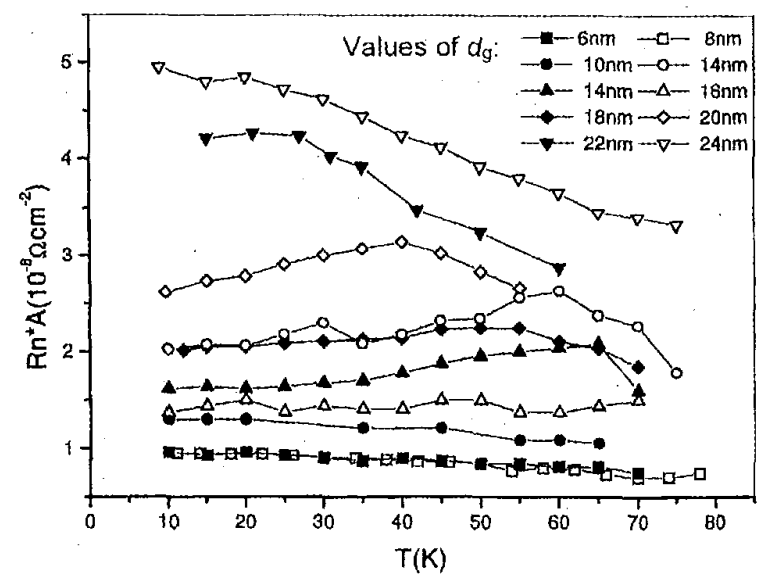

Fig. 5 The temperature dependence of the product $R_{0} d$ for junctions with different values of $d_{t}$ 


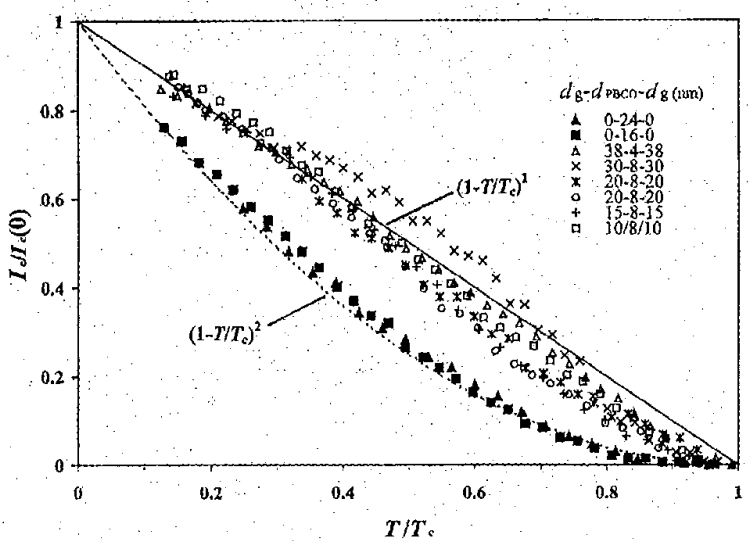

Fig. 6 The normalized $I_{\mathrm{c}}$ vs. $T T_{\mathrm{c}}$ for various $d_{\mathrm{b}}$. The solid symbols are data measured on the junctions with a conventional single barrier of $\mathrm{PBCO}$.

junction. $[1,8]$ The effective barrier thickness for junction $(10-8-10)$ is nearly the same as that with a single barrier $(0-16-0)$, but their $I_{0}(T)$ dependences are far different. The power of two is characteristic of a SNS Junction. The crilical current for a SNS structure can be described by [13]

$$
I_{\mathrm{c}}(T)=C\left\{\left.F_{0}(T)\right|^{2}\left\{\xi_{\mathrm{b}}(T) / \xi_{\mathrm{il}}(T)\right]^{2} \exp \left[-L / \xi_{\mathrm{n}}(T)\right]\right.
$$

where $C$ is a temperature dependent constant, $\xi_{G L}$ the Ginzburg-Landau coherence length, $\xi_{\mathrm{n}}$ the effective coherence length in the normal metal, $F_{0}$ the amplitude of Cooper-pairs in electrodes and $L$ the length of the normal metal path from one superconducting electrode to the other. As $\xi_{\mathrm{GL}}$ and $F_{0}(T)$ vary approximately as $\left(1-T / T_{\mathrm{c}}\right)^{-1 / 2}$ and $\left(1-T / T_{\mathrm{c}}\right)^{1 / 2}$ for $T / T_{\mathrm{c}} \geq 0.5$ and $\xi_{\mathrm{n}}$ varies only slowly, the critical current $I_{\mathrm{c}}$ is proportional to $\left(1-T / T_{i}\right)^{2}$. The fact that the $I_{c}(T)$ of junctions with a continually graded barrier varies as $\left(1-T / T_{c}\right)^{1}$ implies that their transport process might differ from that of the SNS structure. A delailed study of the behavior of these junctions will be discussed elsewhere. [14]

\section{CONCLUSIONS}

In summary, a new barrier structure with a continually graded $\mathrm{Y}_{\mathrm{X}} \mathrm{Pr}_{1-\mathrm{x}} \mathrm{BCO}$ has been prepared by using a simple composite target technique. In such a barrier structure the structural interfaces are absent and the losephson coupling takes place at the electronically formed interfaces. The effective thickness of such a barrier strongly depends on the concentration gradient and temperature. The transport process in these junctions significantly differs from that of junctions with an ordinary single barrier layer and provides a very interesting system for further study. The absence of the structural interface in the weak link region can greatly help to enhance the reproducibility and performance of these junctions.

\section{REFERENCES}

[1] J. Gaw, W.A.M. Aarnink, G.I. Gerrisma, and H. Rogalle, 'Controlled preparation of all high-Te SNS-ts'pe edge junctions and DC SQUDs" Physica C, vol.171, pp.126-130, 1990.

[2] D.K. Chin, and T. Van Duzer,' Novel all-high Tc epitaxial Josephon junction', Appl. Plyys. Lett., vol. 58, pp.753-755, 1941.

[3] B.D. Hunt, M.C. Foote, and L.J. Bajuk, All high Te edge-geometry weak links utilizing Y-Ba-Ci-O barriet layers' Appl. Phys. Lett. vol.59, pp. $982-984,1941$

[4] G. Koren, E. Aharoni, E. Polturak and D. Cohen, 'Properties of all $\mathrm{YBa}_{2} \mathrm{Cu}_{3} \mathrm{O}_{7}$ Josephson edge junctions prepared by in situ laser ablation deposition', Appl. Phys. Lett, vol, 58, pp.634-636, 1991.

[5] J.G. Wen, N. Koshizuka, C. Trwholt, H.W. Zandbergen, E.M.C.M. Reuvekamp, and H. Rogalla, Microstructure of ramp-edge $\mathrm{YBa}_{2} \mathrm{Cu}_{3} \mathrm{O}_{2} / \mathrm{P}_{1} \mathrm{Ba}_{2} \mathrm{Cu}_{3} \mathrm{O}_{\mathrm{N}} / \mathrm{PBB}_{2} \mathrm{Cu}_{3} \mathrm{O}_{\mathrm{x}}$. Iosephson junctions on different substrates', Physica C vol.255, pp.293-305, 1995.

[6] Y. Yang, J. Gao, J.L. Sm. T.C. Chui, and L. Lj, Microstructural study of ramp type multilayer Josephson junetions with different ramp slopes", Physics C vol.300, pp.151-159, 1998.

[7] M.1. Faley, Li. Poppe, H. Solner, C.L. Jia, M. Siegel, and K. Urban, Josephson junctions, interconnects, and crossovers on chemically etched edges of $\mathrm{YBa}_{2} \mathrm{Cu}_{3} \mathrm{O}_{7} \times \mathrm{x}^{\prime}$, Appl. Phys. Lett.,vol.63, 2138-2140, 1993.

[8] .T. Cho, Yu. Boguslavskij, B.B.CY. Klopm:un, D. Tempstra, R. Wijbrans, Q.J. Gerritsma, and $\mathrm{H}$. Rogalla, ' $\mathrm{YBa}_{3} \mathrm{Cu}_{3} \mathrm{O}_{4} / \mathrm{PrBa}_{2} \mathrm{Cu}_{3} \mathrm{O}_{x} / \mathrm{YBa}_{2} \mathrm{Cu}_{3} \mathrm{O}_{x}$ Josephson ramp junctions' J. Appl. Phys, vol.72, pp.575-583, 1992.

[9] O.X. Jia, X.D. Wu, D. Reager, S.R. Folyn, C. Mombourquette, P. Tiwari, I.H. Campbelt, R.J. Houlton, and D.E. Peterson, 'High-temperature superconductor Josephson junctions with a gradient Pr-doped $\mathrm{Y}_{1-\mathrm{X}} \mathrm{Pr}_{\mathrm{x}} \mathrm{Ba}_{2} \mathrm{Cu}_{3} \mathrm{O}_{7-\mathrm{d}}(\mathrm{X} \cdots(0,1,0.3,0.5)$ as batriers", Appl. Phys. Lett. vol.65, pp.2866-2868, 1994

[10] Yu.M. Boguslavskii, I, Gwo, D. Terpstra, G.J. Gerritsma and H. Rogalla, 'Transpolt properties in YBCO/PBCO/YBCO ramp losephson junctions', Physica C. vol. 194, pp.268, 1992.

[11] M.A.J. Verhoeven, O.J. Gerritsma, and H. Rogalla: 'Ramp type HTS Josephson junctions witl PrBaCuGaO barriers, IEEE Tuans. Appl. Supeccon. vol.5, pp. 2005-2098, 1995

[12] W.H. Tang, and J. Gizo, 'Comparison of $T_{0}$-depression of $\mathrm{gr}$ at $\mathrm{Y}$ - and Balsites in $\mathrm{YBa}_{2} \mathrm{Cu}_{3} \mathrm{O}_{y}$ ', Physica C vol.315, pp.59-65 (1999).

[13] 1. Clarke, proc. Roy. Sox. A208, 447 (1969).

[14] J. Gao, and J.L. Sun, 'The stationary properties in YBCO/PrBCO/YBCO ramp junctions with a graded barrier layer' to be published in Physica C. 